\title{
MAXIMAL NON-JAFFARD SUBRINGS OF A FIELD
}

\author{
Mabrouk Ben NasR And NoÔman Jarboui
}

\begin{abstract}
A domain $R$ is called a maximal non-Jaffard subring of a field $L$ if $R \subset L, R$ is not a Jaffard domain and each domain $T$ such that $R \subset T \subset L$ is Jaffard. We show that maximal non-Jaffard subrings $R$ of a field $L$ are the integrally closed pseudo-valuation domains satisfying $\operatorname{dim}_{v} R=\operatorname{dim} R+1$. Further characterizations are given. Maximal non-universally catenarian subrings of their quotient fields are also studied. It is proved that this class of domains coincides with the previous class when $R$ is integrally closed. Moreover, these domains are characterized in terms of the altitude formula in case $R$ is not integrally closed. An example of a maximal non-universally catenarian subring of its quotient field which is not integrally closed is given (Example 4.2). Other results and applications are also given.
\end{abstract}

\section{Introduction}

Throughout this paper, $R \hookrightarrow S$ denotes an extension of commutative integral domains, $\mathrm{gf}(R)$ the quotient field of an integral domain $R, R^{\prime}$ the integral closure of $R$ in its quotient field, and tr. $\operatorname{deg}[S: R]$ the transcendence degree of $\mathrm{qf}(S)$ over $\mathrm{qf}(R)$. If $\operatorname{tr} \operatorname{deg}[S: R]=0$, we say as in [4] that $S$ is algebraic over $R$. We recall that a ring $R$ of finite (Krull) dimension $n$ is a Jaffard ring if its valuative dimension (the limit of the sequence $\left.\left(\operatorname{dim} R\left[X_{1}, \ldots, X_{n}\right]-n, n \in \mathbf{N}\right)\right) \operatorname{dim}_{v} R$, is also $n$. Prüfer domains and Noetherian domains are Jaffard domains. The notion of Jaffard ring is not a local property and thus we say that $R$ is a locally Jaffard ring if $R_{P}$ is a Jaffard ring for each prime ideal $P$ of $R$. We assume familiarity with these concepts as in $[\mathbf{1}],[\mathbf{6}],[\mathbf{1 4}]$.

1991 Mathematics Subject Classification. Primary 13B02; Secondary 13C15, 13A17, 13A18, 13B25, 13E05.

Key words. Jaffard domain, Krull dimension, valuation domain, strong $S$-domain, pullbacks. 
A. R. Wadsworth in [22] considered pairs of domains $R \subset S$ such that each domain $T$ between $R$ and $S$ (that is $R \subseteq T \subseteq S$ ) is Noetherian. In [21], S. Visweswaran noticed that if $S=k\left[y_{1}, \ldots, y_{t}\right]$ is an affine domain (where $k$ is a field) having Krull dimension $n>0$ and if $I$ is a nonzero proper ideal of $S$ and $D$ a subring of $k$, then the $\operatorname{ring} R=D+I$ may be not Noetherian, but each ring $T$ such that $R \subset T \subseteq S$ is Noetherian. In view of this, he introduced the following definition: "Let $A$ be a subring of a Noetherian ring $B$. Then $A$ is said to be a maximal nonNoetherian subring of $B$ if $A$ is non-Noetherian and any subring of $B$ that properly contains $A$ is Noetherian". S. Visweswaran characterized when $D+I$ is a maximal non-Noetherian subring of $k\left[y_{1}, \ldots, y_{t}\right]$ ([21, Proposition 2.1]). On the other hand, A. Ayache and P.-J. Cahen in $[4]$ studied the domains $R$ such that each domain contained between $R$ and its quotient field $\mathrm{qf}(R)$ is Jaffard; that is, the domains whose integral closure $R^{\prime}$ is a Prüfer domain ([4, Théorème 2.6]). They are said to be domains satisfying absolutely the altitude inequality formula. Our purpose here is to complete this circle of ideas by dealing with maximal non-Jaffard subrings of a field $L$; that is, the domains $R$ where $R$ is a nonJaffard domain and each $\operatorname{ring} T, R \subset T \subseteq L$ is Jaffard. First, we show that if $R$ is a maximal non-Jaffard subring of a field $L$, then $L=\operatorname{qf}(R)$. Hence, we may restrict ourselves to the case where $L=\mathrm{qf}(R)$.

Our concern in Section 1 is primarily with maximal non-Jaffard subrings. Our initial line of inquiry was suggested by pointing out a relationship between this kind of domains and pseudo-valuation domains (for short PVDs), which are closely related to valuation domains and have been deeply studied by several authors, notably J. R. Hedstrom, E. G. Houston [17], D. E. Dobbs and M. Fontana [13]. The main result of Section 1 is Theorem 1.4, which states that $R$ is a maximal nonJaffard subring of $\mathrm{qf}(R)$ if and only if $R$ is an integrally closed PVD and $\operatorname{dim}_{v} R=\operatorname{dim} R+1$. Among the several interesting consequences ot this theorem, we only point out that if $R$ is a maximal non-Jaffard subring of $\mathrm{qf}(R)$, then $R$ is a residually integrally closed domain (in the sense that $R / P$ is integrally closed for each prime ideal $P$ of $R$ ). The last part of this section is devoted to a deeper study of these rings, for instance, Theorem 1.7 states that $R$ is a maximal non-Jaffard subring of $\mathrm{qf}(R)$ if and only if $R$ is a local non-Jaffard domain with nonzero maximal ideal $M$ and for each $\operatorname{ring} T$ such that $R \subset T \subseteq \mathrm{qf}(R)$ and for each prime ideal $Q$ of $T$, if $Q \neq M$, then $R /(Q \cap R) \subset T / Q$ is an algebraic extension, and if $Q=M$, then tr. $\operatorname{deg}[T / M: R / M]=1$. If $\mathcal{P}$ is a property which may be possessed by rings (extensions) such as locally (totally) Jaffard, (stably) strong $S$, universally catenarian, we say that 
$R$ is a maximal non- $\mathcal{P}$ subring of a field $L$, if $R$ is a non- $\mathcal{P}$ domain and each ring $T$ such as $R \subset T \subseteq L$ is $\mathcal{P}$. We prove a result analogous to [6, Théorème 5.1]; we show in Theorem 1.8 that the following statements are equivalent:

(i) $R$ is a maximal non-Jaffard subring of qf $(R)$;

(ii) $R$ is a maximal non-locally Jaffard subring of $\mathrm{qf}(R)$;

(iii) $R$ is a maximal non-totally Jaffard subring of $\mathrm{qf}(R)$;

(iv) $R$ is a maximal non-strong $S$ subring of $\mathrm{qf}(R)$;

(v) $R$ is a maximal non-stably strong $S$ subring of $\mathrm{qf}(R)$.

Recall from [4] that a domain $R$ satisfies absolutely the altitude formula; if each overring of $R$ satisfies the altitude formula, equivalently, each overring of $R$ is universally catenarian. Of course these domains satisfy absolutely the altitude inequality formula. However, the converse is not true [4, Exemple 5.1]. Section 2 is concluded with the study of maximal non-universally catenarian subrings of their quotient field. We begin by showing that this class of rings is larger than the class of domains which are maximal non-Jaffard subrings of their quotient fields; and if $R$ is assumed to be integrally closed, then these two classes of domains coincide. As Example 4.2 reveals, Theorem 2.3 is the best possible. This example depends ultimately on the pullback techniques in [15]. It points out the importance of the "integrally closed" hypothesis in Proposition 2.2 and suggests the need of characterizing the maximal non-universally catenarian subrings $R$, when $R$ is not integrally closed. We prove the following (Theorem 2.3):

Let $R$ be a non integrally closed domain. Then $R$ is a maximal nonuniversally catenarian subring of $\mathrm{qf}(R)$ if and only if $R^{\prime}$ is a Prüfer domain, $R$ does not satisfy the altitude formula, and the extension $T \subseteq$ $T^{\prime}$ satisfies the altitude formula for each proper overring $T$ of $R$.

Section 3 finds necessary and sufficient conditions for certain pullbacks to be maximal non-Jaffard (resp., non-universally catenarian) subrings of their quotient fields. Section 4 is devoted to the investigation of several examples illuminating the earlier sections' results, which in some cases are shown to be the best-possible.

It cannot be expected that the typical reader is conversant with all this article's references. Therefore in order to shorten this introduction, we have chosen to recall relevant definitions and facts as needed throughout the article. Any unexplained material is standard as in $[\mathbf{1 6}],[\mathbf{1 9}]$. 


\section{Maximal non-Jaffard subrings of a field}

Let $R$ be a domain contained in a field $L$. We say that $R$ is a maximal non-Jaffard subring of $L$ if $R$ is not Jaffard and each ring $T$ such that $R \subset T \subseteq L$, is Jaffard.

First of all, we establish the following.

Proposition 1.1. Let $R$ be a domain and $L$ a field containing $R$. If $R$ is a maximal non-Jaffard subring of $L$, then $L=\mathrm{qf}(R)$.

Proof: First notice that $L$ is algebraic over $R$. Indeed, if not then there exists an element $X$ of $L$ trancendental over $R$. Hence each overring of $R[X]$ became Jaffard, that is $R^{\prime}[X]$ is a Prüfer domain, which is impossible [4, Théorème 2.6]. Now our task is to prove that $\mathrm{qf}(R)=L$. Assume that $\mathrm{qf}(R) \subset L$ and let $\alpha \in L \backslash \mathrm{qf}(R)$. Then $\alpha$ is algebraic over $R$. Thus there exists an element $r \in R$ such that $r \alpha$ is integral over $R$. Thus $R \subset R[r \alpha]$ is an integral extension. But $R[r \alpha]$ is a Jaffard domain. Hence, according to [1, Proposition 1.1], $R$ is a Jaffard domain, the desired contradiction to complete the proof.

As a direct consequence of Proposition 1.1, the study of maximal nonJaffard subrings of a field $L$ can be reduced to the case where $L=\mathrm{qf}(R)$.

Now, notice that if $R$ is a maximal non-Jaffard subring of its quotient field, then $R$ is integrally closed. Indeed, if $R \neq R^{\prime}$, then $R^{\prime}$ is a Jaffard domain, and hence so is $R$ (since $R \subset R^{\prime}$ is an integral extension [1, Proposition 1.1]) which is impossible.

In this section, we collect more information on this kind of domains and we characterize them in terms of pseudo-valuation domains. We begin by presenting some terminology. Let $B$ be an integral domain, $I$ an ideal of $B$, and $D$ a subring of $B / I$. Consider the pullback construction of commutative rings:

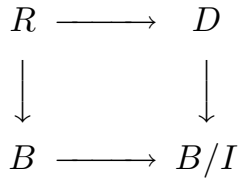

Following [9], we say that $R$ is the domain of the $(B, I, D)$ construction and we set $R:=(B, I, D)$. Next we consider the case where $I$ is assumed to be maximal. Denoting by $M$ the ideal $I, T$ the domain $B, K$ the field $T / M$, and $\varphi: T \longrightarrow K$ the natural epimorphism. 
We next recall a few wellknown properties about pullbacks to be used in our next theorems and examples. First, $M$ is a common ideal of both $R$ and $T, M=(R: T)=\{x \in T \mid x T \subset R\}$, and $R / M \simeq D$. For each $P \in \operatorname{Spec}(R)$ with $M \subseteq P$, there is a (unique) $Q \in \operatorname{Spec}(D)$ such that $\varphi^{-1}(Q)=P$; and $\varphi^{-1}\left(D_{Q}\right)=R_{P}$. If $T$ is local, then each prime ideal of $R$ compares with $M$, and thus $\operatorname{dim} R=\operatorname{dim} D+\operatorname{dim} T$. Also $R$ is local if and only if both $D$ and $T$ are local (cf. [15, Theorem 1.4, Proposition 2.1]).

The last construction to be noted here concerns the notion of a pseudovaluation domain (for short, a PVD), which was introduced by Hedstrom and Houston $[\mathbf{1 7}]$ and has been studied subsequently in $[\mathbf{2}],[\mathbf{1 1}],[\mathbf{1 2}]$ and [18]. A domain $R$ is said to be a PVD in case each prime ideal $P$ of $R$ is strongly prime, in the sense that whenever $x, y \in \mathrm{qf}(R)$ satisfy $x y \in P$, then either $x \in P$ or $y \in P$, equivalently, in case $R$ has a (uniquely determined) valuation overring $V$ such that $\operatorname{Spec}(R)=\operatorname{Spec}(V)$ as sets, equivalently (by [2, Proposition 2.6]) in case $R$ is a pullback of the form $V \times{ }_{K} k$ where $V$ is a valuation domain with residue field $K$ and $k$ is a subfield of $K$. As the terminology suggests, any valuation domain is a PVD $[\mathbf{1 7}$, Proposition 1.1]. Although the converse is false [17, Example 2.1], any PVD must, at least, be local [17, Corollary 1.3].

Before stating Theorem 1.4, we establish a lemma which serves both to motivate this theorem and to dispatch the difficult implication in its proof. First it is convenient to recall that given a ring extension $R \subset S$, $(R, S)$ is said to be a Jaffard pair [7], if each $\operatorname{ring} T$ between $R$ and $S$ is Jaffard.

Lemma 1.2. Let $k \subset K$ be an extension of fields. Then the following statements are equivalent.

(i) $(k, K)$ is a Jaffard pair;

(ii) tr. $\operatorname{deg}[K: k] \leq 1$.

Proof: (ii) $\Rightarrow$ (i) Assume that tr. $\operatorname{deg}[K: k] \leq 1$ and let $T$ be a ring between $k$ and $K$. We have $\operatorname{dim}_{v} T \leq \operatorname{dim}_{v} k+\operatorname{tr} \cdot \operatorname{deg}[T: k]$ (cf. [4, Lemme 1.1]). Thus $\operatorname{dim}_{v} T \leq 1$. If $T$ is not a field, then $\operatorname{dim} T=$ $\operatorname{dim}_{v} T=1$, so $T$ is a Jaffard domain.

(i) $\Rightarrow$ (ii) If tr. $\operatorname{deg}[K: k] \geq 2$, let $X, Y$ be two transcendental algebraically independent elements of $K$ over $k$. Then, the domain $T=$ $k+Y k(X)[Y]_{(Y)}$ is contained between $k$ and $K$, and we have $\operatorname{dim} T=1$ and $\operatorname{dim}_{v} T=2$ [1, Proposition 2.5]. Thus $T$ is not a Jaffard domain, which contradicts assertion (i). Hence tr. $\operatorname{deg}[K: k] \leq 1$.

Now we establish the following usefull result. 
Lemma 1.3. Let $V$ be a valuation domain with maximal ideal $M$ and residue field $K$. Let $D$ be a subring of $K$ and $R:=(V, M, D)$. Then the overrings of $R$ are exactly the overrings of $V$ and the intermediate domains between $R$ and $V$.

Proof: Let $S$ be an overring of $R$ such that $V \nsubseteq S$. Thus there exists $v \in V$ and $v \notin S$. Our task is to show that $S \subseteq V$. Let $s \in S$, assume that $s \notin V$. Since $V$ is a valuation domain, then $\frac{1}{s} \in M$. Hence $\frac{v}{s} \in M$. Therefore $v=\frac{v}{s} s \in S$, the desired contradiction.

As Lemma 1.3 is to be used repeatedly in the proof of some of the next theorems, we make use of it without any reference or comment.

Theorem 1.4. Let $R$ be a domain. Then the following statements are equivalent:

(i) $R$ is a maximal non-Jaffard subring of $\mathrm{qf}(R)$;

(ii) $R$ is an integrally closed $P V D$ with maximal ideal $M$ and tr. $\operatorname{deg}[V / M: R / M]=1$, where $V$ is the associated valuation domain of $R$;

(iii) $R$ is an integrally closed $P V D$ and $\operatorname{dim}_{v} R=\operatorname{dim} R+1$.

Proof: (i) $\Rightarrow$ (ii) We have already observed that $R$ is integrally closed. Now we claim that there exists a valuation overring $V$ of $R$ and a nonzero prime ideal $Q$ of $V$ such that $R=R_{P}+Q V_{Q}$, where $P=Q \cap R$. Indeed, assume that $R \subset R_{P}+Q V_{Q}$ for each valuation overring $V$ of $R$ and each nonzero prime ideal $Q$ of $V$. Then $R_{P}+Q V_{Q}$ is a Jaffard domain. Hence $R \subset V$ is a residually algebraic extension [1, Proposition 2.5 (b)]. Thus $R^{\prime}$ is a Prüfer domain ([16, Theorem 19.15]), and therefore $R$ is a Jaffard domain. Now $R=R_{P}+Q V_{Q}$ for some valuation overring $V$ of $R$ and nonzero prime ideal $Q$ of $V$. Thus $R:=\left(V_{Q}, Q V_{Q}, R_{P} / P R_{P}\right)$. Hence $R$ is a PVD (cf. [2]).

Denote $Q V_{Q}$ by $M$. Then $M$ is the unique maximal ideal of $R$. Notice that $M=M \cap R=Q V_{Q} \cap R=Q \cap R=P$. Our task is to show that tr. $\operatorname{deg}[V / M: R / M]=1$. Let $D$ be a domain such that $R / M \subset D \subset$ $V / M$ and consider the ring $T:=(V, M, D)$. Then $R \subset T \subset V$, so $T$ is a Jaffard domain. Hence $D$ is a Jaffard domain $[\mathbf{1}$, Theorem 2.6]. Thus each intermediate domain between $R / M$ and $V / M$ is Jaffard. Hence by Lemma 1.2, we get tr. $\operatorname{deg}[V / M: R / M] \leq 1$. The extension $R / M \subset V / M$ can not be algebraic since $R$ is not Jaffard [1, Proposition $2.5(\mathrm{~b})]$. Thus tr. $\operatorname{deg}[V / M: R / M]=1$. 
(ii) $\Rightarrow$ (i) It is clear that $R$ is not a Jaffard domain $[\mathbf{1}$, Proposition 2.5 (b)]. Now, let $T$ be a domain such that $R \subset T \subseteq \mathrm{qf}(R)$. Then either $T$ is an overring of $V$, so it is Jaffard, or $T$ is an intermediate domain between $R$ and $V$, so $T:=(V, M, D)$, where $R / M \subset D \subseteq V / M$. According to Lemma $1.2, D$ is a Jaffard domain, and since $R$ is integrally closed, then tr. $\operatorname{deg}[V / M: D]=0$. Thus $T$ is a Jaffard domain [1, Theorem 2.6]. Hence $R$ is a maximal non-Jaffard subring of $\mathrm{qf}(R)$.

(ii) $\Leftrightarrow$ (iii) We have $\operatorname{dim} R=\operatorname{dim} V$ and $\operatorname{dim}_{v} R=\operatorname{dim} V+$ tr. $\operatorname{deg}[V / M$ : $R / M$ ] (cf. [1, Proposition 2.5 (a)]). Thus $\operatorname{dim}_{v} R=\operatorname{dim} R+1$ if and only if tr. $\operatorname{deg}[V / M: R / M]=1$.

We turn now to point out the connection with integrally closed domains. A special result is that if $R$ is a maximal non-Jaffard subring of qf $(R)$, then $R / P$ is integrally closed for each prime ideal $P$ of $R$. We begin by noticing that if $R$ is a maximal non-Jaffard subring of qf $(R)$, then for each non-maximal prime ideal $P$ of $R, R_{P}$ is a valuation domain since $V \subset R_{P}$. Nevertheless we have the following:

Proposition 1.5. Let $R$ be a maximal non-Jaffard subring of $\mathrm{qf}(R)$. Then for each non maximal prime ideal $P$ of $R, R / P$ is a maximal non-Jaffard surbring of its quotient field.

Proof: Notice that $R / P$ is not a Jaffard domain. Indeed, consider the domain $R+P R_{P}$. If $R / P$ is a Jaffard domain, then so is $R+P R_{P}$ $[\mathbf{1}$, Theorem $2.6(\mathrm{~b})]$. Thus $R \subset R+P R_{P}$. But $R$ is a PVD, so $R:=$ $(V, M, k)([\mathbf{2}])$, where $V$ is the associated valuation domain and $k=$ $R / M$. Since $P \subset M$, then there exists a prime ideal $Q$ of $V$ such that $R_{P}=V_{Q}$. Thus $P R_{P}=Q V_{Q}=Q \subseteq M$. Therefore $R+P R_{P}=$ $R+Q \subseteq R+M=R$. This contradicts the fact that $R \subset R+P R_{P}$. Consequently $R / P$ is not a Jaffard domain. Now, let $D$ be a domain such that $R / P \subset D \subseteq \mathrm{qf}(R / P)$ and consider the $\operatorname{ring} T:=\left(R_{P}, P R_{P}, D\right)$. Since $R / P \subset D \subseteq \mathrm{qf}(R / P)$, then $R \subset T \subseteq \mathrm{qf}(R)$. Hence $T$ is a Jaffard domain, and thus so is $D[\mathbf{1}$, Theorem 2.6]. Therefore $R / P$ is a maximal non-Jaffard subring of its quotient field.

Remark 1.6. By the previous proposition, we conclude that if $R$ is a maximal non-Jaffard subring of $\mathrm{qf}(R)$, then $R / P$ is integrally closed for each prime ideal $P$ of $R$. The converse does not hold. More precisely, in Example 4.1 we construct for each $n, m \in \mathbf{N}$ such that $n+1 \leq m \leq$ $2 n+1, n \geq 2$ a residually integrally closed local domain $R$ such that $\operatorname{dim} R=n, \operatorname{dim} R[X]=m$, and $R$ is not a maximal non-Jaffard subring of its quotient field. 
The next result provides another characterization of a domain $R$ which is a maximal non-Jaffard subring of its quotient field.

Theorem 1.7. Let $R$ be domain. Then the following statements are equivalent:

(i) $R$ is a maximal non-Jaffard subring of $\mathrm{qf}(R)$;

(ii) $R$ is local non-Jaffard domain with nonzero maximal ideal $M$, and for each ring $T$ such that $R \subset T \subseteq \mathrm{qf}(R)$ and for each $Q \in \operatorname{Spec}(T):$ If $Q \neq M$, then $R /(Q \cap R) \subset T / Q$ is algebraic, and if $Q=M$, then $\operatorname{tr} \cdot \operatorname{deg}[T / M: R / M]=1$.

Proof: (i) $\Rightarrow$ (ii) By Theorem 1.4, $R$ is local since it is a PVD. Now let $T$ be a domain such that $R \subset T \subseteq \mathrm{qf}(R)$. Then either $T$ is an overring of $V$, where $V$ is the associated valuation domain of $R$ or $T$ is an intermediate domain between $R$ and $V$. In the first case, let $Q \in \operatorname{Spec}(T)$ such that $Q \neq M$, then $R /(Q \cap R) \subset V /(Q \cap V) \subset T / Q$. But tr. $\operatorname{deg}[V /(Q \cap V)$ : $R /(Q \cap R)]=0$ since $Q \cap R \neq M$. Hence tr. $\operatorname{deg}[T / Q: R /(Q \cap R)]=$ tr. $\operatorname{deg}[T / Q: V /(Q \cap V)]+$ tr. $\operatorname{deg}[V /(Q \cap V): R /(Q \cap R)]=0$. Now, if $Q=M$, then $T=V$. Hence tr. $\operatorname{deg}[T / M: R / M]=\operatorname{tr} \cdot \operatorname{deg}[V / M:$ $R / M]=1$ (by Theorem 1.4). In the second case, $T$ shares the ideal $M$ with $R$ and $V$. If $Q \in \operatorname{Spec}(T)$ and $Q \neq M$, then we discuss two cases:

Case 1: If $M \nsubseteq Q$, then $T_{Q}=R_{Q \cap R}$, so $R /(Q \cap R) \subset T / Q$ is algebraic.

Case 2: If $M \subset Q$, then $R+Q T_{Q} \neq R$, so $R+Q T_{Q}$ is a Jaffard domain. Thus $R /(Q \cap R) \subset T / Q$ is an algebraic extension ([1, Theorem 2.6]).

Now, if $Q=M$, then tr. $\operatorname{deg}[T / M: R / M]=\operatorname{tr} . \operatorname{deg}[V / M: R / M]-$ tr. $\operatorname{deg}[V / M: T / M]=1$.

(ii) $\Rightarrow$ (i) Let $T$ be a domain such that $R \subset T \subseteq$ qf $(R)$, and let $S$ be an overring of $T$. If $Q \in \operatorname{Spec}(S)$, then either $Q \neq M$, so by hypothesis, we have tr. $\operatorname{deg}[S / Q: R /(Q \cap R)]=0$. Thus tr. $\operatorname{deg}[S / Q: T /(Q \cap T)]=0$. Or $Q=M$, so tr. $\operatorname{deg}[S / M: R / M]=1$. Hence tr. $\operatorname{deg}[S / M: T / M]=$ tr. $\operatorname{deg}[S / M: R / M]-\operatorname{tr} \cdot \operatorname{deg}[T / M: R / M]=0$. Therefore $T \subseteq S$ is a residually algebraic extension for each overring $S$ of $T$. Thus $T$ satisfies absolutely the altitude inequality formula [4, Théorème 2.6]. In particular, $T$ is a Jaffard domain. Thus $R$ is a maximal non-Jaffard subring of $\mathrm{qf}(R)$.

Recall that a ring $R$ is said to be a strong $S$-domain if for each pair of consecutive prime ideals $P \subset Q$ of $R$, the extended primes $P[X] \subset$ $Q[X]$ are consecutive. The most natural examples of strong $S$-domains are arbitrary Noetherian domains $[\mathbf{1 9}$, Theorem 68$]$. Dispite the above 
material, the class of strong $S$-domains is not very stable, for instance with respect to polynomial extensions. Following [20], we say that $R$ is a stably strong $S$-domain if $R\left[X_{1}, \ldots, X_{n}\right]$ is a strong $S$-domain for each nonnegative integer $n$.

A domain $R$ is said to be totally Jaffard if $R / P$ is a locally Jaffard domain for each prime ideal $P$ of $R$ (cf. [10]).

We establish the following result.

Theorem 1.8. Let $R$ be a domain. Then the following statements are equivalent:

(i) $R$ is a maximal non-Jaffard subring of $\mathrm{qf}(R)$;

(ii) $R$ is a maximal non-locally Jaffard subring of $\mathrm{qf}(R)$;

(iii) $R$ is a maximal non-totally Jaffard subring of $\mathrm{qf}(R)$;

(iv) $R$ is a maximal non-strong $S$ subring of $\mathrm{qf}(R)$;

(v) $R$ is a maximal non-stably strong $S$ subring of $\mathrm{qf}(R)$.

Proof: It follows readily from [6, Théorème 5.1].

\section{Maximal non-universally catenarian subrings of their quotient fields}

First recall that an extension $R \subseteq S$ of integral domains satisfies the altitude inequality formula (resp., the altitude formula), if for each prime ideal $Q$ of $S$, if we set $P=Q \cap R$, we have $h t Q+\operatorname{tr} \cdot \operatorname{deg}[S / Q: R / P] \leq$ $h t P+\operatorname{tr} . \operatorname{deg}[S: R]$ (resp., $h t Q+\operatorname{tr} \cdot \operatorname{deg}[S / Q: R / P]=h t P+\operatorname{tr} \cdot \operatorname{deg}[S:$ $R]$ ). A domain $R$ is said to satisfy the altitude inequality formula (resp., the altitude formula) if $R \subseteq S$ satisfies the altitude inequality formula (resp., the altitude formula) for each finite type $R$-algebra $S$ containing $R$.

A ring $R$ is said to be catenarian in case, for each pair $P \subset Q$ of prime ideals of $R$, all saturated chains of primes from $P$ to $Q$ have a common finite length. We shall say that $R$ is universally catenarian if the polynomial rings $R\left[X_{1}, \ldots, X_{n}\right]$ are catenarian for each positive integer $n[\mathbf{8}]$. Notice that if $R$ is universally catenarian, then it satisfies the altitude formula $[\mathbf{8}$, Theorem 5.1].

In [4], A. Ayache and P.-J. Cahen studied the domains $R$ such that each domain $T$ between $R$ and qf $(R)$ satisfies the altitude formula. These domains are said to satisfy absolutely the altitude formula. They established that $R$ satisfies absolutely the altitude formula if and only if $R^{\prime}$ is a Prüfer domain and $R \subseteq R^{\prime}$ satisfies the altitude formula ([4, Théorème 3.3]). Naturally these domains satisfy absolutely the altitude inequality formula, but the converse does not hold (cf. [4, Exemple 5.1]). 
We say that a domain $R$ is a maximal subring of $\mathrm{qf}(R)$ not satisfying the altitude formula, if $R$ does not satisfy the altitude formula and each domain $T$ such that $R \subset T \subseteq \mathrm{qf}(R)$ satisfies the altitude formula. We show in the following that if $R$ is a maximal non-Jaffard subring of qf $(R)$, then it is maximal not satisfying the altitude formula and the converse is not true (see Example 4.2). But first a key lemma:

Lemma 2.1. Let $R$ be a maximal subring of $\mathrm{qf}(R)$ not satisfying the altitude formula. Then the following statements are equivalent:

(i) $R$ is integrally closed;

(ii) $R$ is not a Jaffard domain;

(iii) The extension $R \subseteq R^{\prime}$ satisfies the altitude formula.

Proof: (i) $\Rightarrow($ ii) Assume that $R$ is a Jaffard domain. Since each proper overring of $R$ satisfies the altitude formula, then $R^{\prime}$ is a Prüfer domain ([4, Théorème 2.6]). Hence $R=R^{\prime}$ satisfies the altitude formula, the desired contradiction.

(ii) $\Rightarrow$ (i) If $R$ is not integrally closed, then $R^{\prime}$ satisfies the altitude formula. Hence $R^{\prime}$ is a Jaffard domain and so is $R$ [1, Proposition 1.1], a contradiction.

(i) $\Rightarrow$ (iii) This is trivial.

(iii) $\Rightarrow$ (i) Assume that $R \neq R^{\prime}$. Since $R^{\prime}$ satisfies the altitude formula and $R \subset R^{\prime}$ satisfies the altitude formula, then by [14, Proposition 2.6], $R$ also satisfies the altitude formula. This is the desired contradiction to complete the proof.

Proposition 2.2. Let $R$ be an integrally closed domain. Then the following statements are equivalent:

(i) $R$ is a maximal non-universally catenarian subring of $\mathrm{qf}(R)$;

(ii) $R$ is a maximal subring of $\mathrm{qf}(R)$ not satisfying the altitude formula;

(iii) $R$ is a maximal non-Jaffard subring of $\mathrm{qf}(R)$.

Proof: (i) $\Leftrightarrow$ (ii) It follows readily from [4, Théorème 3.8] without the assumption $R$ is integrally closed.

(ii) $\Rightarrow$ (iii) Each proper overring of $R$ satisfies the altitude formula; hence $R$ is Jaffard. By Lemma $2.1, R$ is not a Jaffard domain. Thus $R$ is a maximal non-Jaffard subring of its quotient field.

(iii) $\Rightarrow$ (i) Since $R$ is not a Jaffard domain, then it is not universally catenarian [8, Corollary 3.3]. By Theorem 1.4, $R$ is a PVD. Thus there exists a valuation overring $V$ of $R$ such that $R:=(V, M, k)$, where $M$ is the maximal ideal of $V$ and $k$ is a subfield of $K=V / M$. Let $T$ be a domain such that $R \subset T \subseteq \operatorname{qf}(R)$. If $T$ is an overring of $V$, 
then it is universally catenarian since it is a valuation domain. If not, $T:=(V, M, D)$, where $D$ is a domain properly contained between $k$ and $K$. We have tr. $\operatorname{deg}[K: D]=0$. Thus $\operatorname{dim}_{v} D \leq \operatorname{tr}$. $\operatorname{deg}[D: k]=1$ (cf. $[4$, Lemme 1.1]). Hence, either $D$ is a field or a one-dimensional Jaffard domain. Therefore by $[8$, Corollary 6.3$], D$ is universally catenarian. According to [3, Corollary 2.3], $T$ is a universally catenarian domain, which completes the proof.

The case when $R$ is not integrally closed will now be given.

Theorem 2.3. Let $R$ be a non integrally closed domain. Then the following statements are equivalent:

(i) $R$ is a maximal non-universally catenarian subring of $\mathrm{qf}(R)$;

(ii) $R^{\prime}$ is a Prüfer domain, $R$ does not satisfy the altitude formula, and $T \subseteq T^{\prime}$ satisfies the altitude fromula for each $T$ such that $R \subset T \subseteq \mathrm{qf}(R)$.

Proof: (i) $\Rightarrow$ (ii) We have $R \neq R^{\prime}$. Then each overring of $R^{\prime}$ is universally catenarian. Thus by [4, Théorème 3.3], $R^{\prime}$ is a Prüfer domain. Now, let $T$ be an overring of $R$ such that $R \neq T$. Then $T$ satisfies absolutely the altitude formula. Hence $T \subseteq T^{\prime}$ satisfies the altitude formula (cf. [4, Théorème 3.3]).

(ii) $\Rightarrow$ (i) Let $T$ be a ring such that $R \subset T \subseteq \mathrm{qf}(R)$. Then $R^{\prime} \subseteq T^{\prime} \subseteq$ $\operatorname{qf}(R)$. Thus $T^{\prime}$ is a Prüfer domain since it is an overring of the Prüfer domain $R^{\prime}$. On the other hand, $T \subseteq T^{\prime}$ satisfies the altitude formula. Thus by [8, Theorem 6.1], $T$ is universally catenarian. Since $R$ does not satisfy the altitude formula, then it is not universally catenarian. Hence $R$ is a maximal non-universally catenarian subring of $\mathrm{qf}(R)$.

\section{Transfer results}

In this section, we begin by determining when a pullback $R$ is a maximal non-Jaffard subring of its quotient field. We recall some notation for conductors. If $R$ is a domain and $I$ and $J$ are $R$-submodules of $\mathrm{qf}(R)$, then $(I: J)=\{x \in \mathrm{qf}(R) \mid x J \subset I\}$. If $R$ is a PVD with associated valuation domain $V$ and maximal ideal $M$, assume that $R \neq V$, then $M$ is not a principal ideal of $R$ and $V=(M: M)[\mathbf{2}$, Proposition 2.3], and by $[\mathbf{2}$, Lemma 2.4], we get $V=(R: M)=(M: M)$.

We establish the following theorem.

Theorem 3.1. Let $T$ be a local domain with maximal ideal $M$, and $D$ a subring of the field $K=T / M$. Let $R:=(T, M, D)$. Then the following hold. 
(a) If $D$ is a field, then $R$ is a maximal non-Jaffard subring of $\mathrm{qf}(R)$ if and only if $T$ is a $P V D$ satisfying absolutely the altitude inequality formula, $D$ is algebraically closed in $(M: M) / M$, and $\operatorname{tr} . \operatorname{deg}[K$ : $D]=1$.

(b) If $D$ is not a field, then $R$ is a maximal non-Jaffard subring of $\mathrm{qf}(R)$ if and only if $T$ is a valuation domain, $D$ is a maximal non-Jaffard subring of its quotient field, and $\mathrm{qf}(D)=K$.

Proof: (a) Assume that $R$ is a maximal non-Jaffard subring of its quotient field. Since $T$ properly contains $R$, then $T$ is a Jaffard domain. Thus $T$ satisfies absolutely the altitude inequality formula (since each proper overring of $T$ is a proper overring of $R$, so it is Jaffard). The ring $R$ is a PVD, so there exists a valuation domain $V$ with $M$ as a maximal ideal such that $R:=(V, M, D)$. Now, $T$ is an overring of $R$, thus $T$ is a $\operatorname{PVD}(\operatorname{since} \operatorname{Spec}(R)=\operatorname{Spec}(T))$. On the other hand, we have:

$$
\begin{aligned}
\operatorname{tr} . \operatorname{deg}[K: D] & =\operatorname{dim}_{v} R-\operatorname{dim}_{v} T & & (\text { cf. }[\mathbf{1}, \text { Proposition 2.5]) } \\
& =(\operatorname{dim} R+1)-\operatorname{dim} T & & (\text { cf. Theorem 1.4) } \\
& =1 & & (\text { since } \operatorname{dim} R=\operatorname{dim} T) .
\end{aligned}
$$

Now, $V=(M: M)$. Since $R$ is integrally closed (Theorem 1.4), then $D$ is algebraically closed in $V / M=(M: M) / M$ (cf. [9, Proposition 2]).

Conversely, $R$ is not a Jaffard domain since $D \subset K$ is not an algebraic extension [1, Proposition 2.5]. The ring $T$ is a PVD, so there is a valuation domain $W$ with maximal ideal $M$ such that $T:=(W, M, K)$. But $R:=(T, M, D)$. Hence $R$ is a PVD with associated valuation domain $W=(M: M)$. Furthermore, $\operatorname{dim}_{v} R-\operatorname{dim} R=\operatorname{dim}_{v} T+\operatorname{tr} \cdot \operatorname{deg}[K:$ $D]-\operatorname{dim} T=\operatorname{tr} \cdot \operatorname{deg}[K: D]=1$. Since $D$ is algebraically closed in $W / M$, then $R$ is integrally closed. Thus by Theorem $1.4, R$ is a maximal non-Jaffard subring of qf $(R)$.

(b) Assume that $R$ is a maximal non-Jaffard subring of its quotient field. Then $R$ is a PVD. Hence there exists a valuation domain $V$ with $m$ as a maximal ideal such that $R:=\left(V, m, k^{\prime}\right)$. We show under the assumption on $D$ that $T$ must be an overring of $V$. Indeed, assume that $R \subset T \subseteq V$, then $T$ shares the ideal $m$ with $R$ and $V$, so $T:=$ $(V, m, T / m)$. Since $T$ is local with $M$ as a maximal ideal, then $m \subseteq M$. On the other hand, we have $M \subseteq m$ (since $R$ is local with maximal ideal $M)$. Thus $m=M$ and $R / M=R / m=D$ is a field, a contradiction. Therefore $T$ is an overring of $V$, so $T$ is a valuation domain. According to Proposition 1.5, the domain $D$ is a maximal non-Jaffard subring of its quotient field. 
Assume now that $\mathrm{qf}(D) \subset K$ and let $a \in K \backslash \mathrm{qf}(D)$. Since tr. $\operatorname{deg}[K$ : $D]=\left(\operatorname{dim}_{v} R-\operatorname{dim} R\right)-\left(\operatorname{dim}_{v} T-\operatorname{dim} T\right)-\left(\operatorname{dim}_{v} D-\operatorname{dim} D\right)=0$, then $a$ is algebraic over qf $(D)$. Hence there exists $d \in D$ such that $a d$ is integral over $D$. The element $a d \notin D$ because $a \notin \operatorname{qf}(D)$. On the other hand, $D \subset D[a d]$ is an integral extension. Hence $D[a d]$ is not a Jaffard domain because $D$ is not a Jaffard domain ([1, Proposition 1.1]). Let $S:=(V, m, D[a d])$. The ring $S$ is such that $R \subset S \subset T$ and $S$ is not a Jaffard domain, which contradicts the fact that $R$ is a maximal nonJaffard subring of its quotient field. Therefore $\mathrm{qf}(D)=K$. Conversely, since $R:=(T, M, D)$ and $T$ is a valuation domain, then each proper overring $S$ of $R$ is either an overring of $T$, hence it is Jaffard or a ring contained between $R$ and $T$, and in the latter case $S:=\left(T, M, D_{1}\right)$, where $D_{1}$ is a ring such that $D \subset D_{1} \subset K=\mathrm{qf}(D)$. Hence $D_{1}$ is a Jaffard domain, and thus so is $S$ ([1, Theorem 2.6]).

We close this section by studying when a pullback domain which is not integrally closed is a maximal non-universally catenarian subring of its quotient field. First we show that such domains are local.

Lemma 3.2. Let $R$ be maximal non-universally catenarian subring of $\mathrm{qf}(R)$. Then $R$ is local.

Proof: The case when $R$ is integrally closed follows from Theorem 1.4 and Proposition 2.2. Suppose that $R$ is not integrally closed. By Lemma 2.1, the extension $R \subset R^{\prime}$ does not satisfy the altitude formula. Thus there exists a prime ideal $Q$ of $R^{\prime}$ such that $h t_{R^{\prime}} Q<h t_{R} P$, where $P=Q \cap R$. Let $N=R \backslash P$. The extension $R_{P} \subset N^{-1} R^{\prime}=\left(R_{P}\right)^{\prime}$ does not satisfy the altitude formula, which implies by Theorem 2.3 (ii) that $R=R_{P}$, as required.

Before presenting our next theorem, we establish the following.

Proposition 3.3. Let $T$ be a local domain with maximal ideal $M, D$ a subring of the field $K=T / M$, and $R:=(T, M, D)$. Assume that $R$ is not integrally closed. If $R$ is a maximal non-universally catenarian subring of $\mathrm{qf}(R)$, then $D$ is a Jaffard domain which is maximal nonuniversally catenarian subring of its quotient field and $T^{\prime}$ is a Prüfer domain.

Proof: The domain $T$ is a proper overring of $R$. Hence each proper overring of $T$ is universally catenarian. Thus by [4, Théorème 3.3], $T^{\prime}$ is a Prüfer domain. By Lemma 2.1, $R$ is a Jaffard domain. Hence $D$ is a Jaffard domain and $D \subset K$ is an algebraic extension (cf. [1, Theorem 2.6]). According to [3, Corollary 2.3], $D$ is not universally 
catenarian. Now, let $D_{1}$ be a domain such that $D \subset D_{1} \subseteq$ qf $(D)$ and set $R_{1}:=\left(T, M, D_{1}\right) . R_{1}$ is a proper overring of $R$, so it is universally catenarian. Hence $D_{1} \simeq R_{1} / M$ is also universally catenarian. This yields that $D$ is a maximal non-universally catenarian subring of its quotient field.

Theorem 3.4. Let $T$ be a Prüfer domain, $M$ a maximal ideal of $T, D$ a subring of the field $K=T / M$, and $R:=(T, M, D)$. Assume that $R$ is not integrally closed. Then the following statements are equivalent:

(i) $R$ is a maximal non-universally catenarian subring of its quotient field;

(ii) $T$ is a valuation domain with maximal ideal $M, D$ is a maximal non-universally catenarian subring of $K$ (that is $D$ is not universally catenarian and each domain $D_{1}$ such that $D \subset D_{1} \subseteq K$ is universally catenarian), and $D$ is a Jaffard domain.

Proof: (i) $\Rightarrow$ (ii) By Lemma 3.2, $R$ is local. Then so is $T$. Thus $T$ is a valuation domain with maximal ideal $M$. On the other hand, the domain $R^{\prime}$ is universally catenarian, in particular it is Jaffard [8, Corollary 3.3]. Hence $R$ is a Jaffard domain [1, Proposition 1.1]. Thus $D \subset K$ is an algebraic extension and $D$ is a Jaffard domain [1, Theorem 2.6]. Since $R$ does not satisfy the altitude formula, then so does $D[\mathbf{5}$, Lemme 3.6].

Now, since $R^{\prime}$ is a Prüfer domain, then so is $D^{\prime}[4$, Proposition 2.2 and Théorème 2.6]. It remains to show that if $D_{1}$ is a ring such that $D \subset D_{1} \subseteq K$, then $D_{1}$ is a universally catenarian domain. For this, $D_{1}^{\prime}$ is universally catenarian since $D^{\prime} \subset D_{1}^{\prime}$ is an algebraic extension ([4, Corollaire 3.9]). By [8, Theorem 6.1], it suffices to show that $D_{1} \subset D_{1}^{\prime}$ satisfies the altitude formula. Let $R_{1}:=\left(T, M, D_{1}\right), R_{2}:=\left(T, M, D_{1}^{\prime}\right)$. It is clear that $R_{1} \subseteq R_{2} \subseteq R_{1}^{\prime}$. The extension $R_{1} \subseteq R_{1}^{\prime}$ satisfies the altitude formula (Theorem 2.3); $R_{1} \subseteq R_{2}$ and $R_{2} \subseteq R_{1}^{\prime}$ satisfy the altitude inequality formula since $R_{1}$ and $R_{2}$ are locally Jaffard domains. $R_{2} \subseteq R_{1}^{\prime}$ is a lying-over extension. Thus by [4, Lemme 3.1], $R_{1} \subseteq R_{2}$ satisfies the altitude formula. Now, let $Q_{1}^{\prime} / M \in \operatorname{Spec}\left(D_{1}^{\prime}\right)$ and set $Q_{1}=Q_{1}^{\prime} \cap R_{1}$. We have $h t_{R_{2}} Q_{1}^{\prime}=h t_{R_{1}} Q_{1}$. Thus $h t_{D_{1}^{\prime}}\left(Q_{1}^{\prime} / M\right)=$ $h t_{R_{2}} Q_{1}^{\prime}-h t_{R_{2}} M=h t_{R_{1}} Q_{1}-h t_{R_{1}} M=h t_{D_{1}}\left(Q_{1} / M\right)$ (because $M$ is a divided prime in $R_{1}$ and $R_{2}$ and hence is comparable with all primes of $R_{1}$ and $R_{2}$ ). Therefore $D_{1} \subset D_{1}^{\prime}$ satisfies the altitude formula.

(ii) $\Rightarrow$ (i) Notice that $D \subseteq K$ is an algebraic extension since $D$ is a maximal non-universally catenarian subring of $K$. Now let $R_{1}$ be a domain such that $R \subset R_{1} \subseteq \mathrm{qf}(R)$. If $R_{1}$ is an overring of $T$, then it is universally catenarian. If not, we get $R \subset R_{1} \subseteq T$. Hence $R_{1}:=\left(T, M, D_{1}\right)$, where $D \subset D_{1} \subseteq K$. Hence $R_{1}$ is universally catenarian [3, Corollary 2.3]. 
Now, since $D$ is Jaffard and it is maximal non-universally catenarian subring of $K$, then $D \neq D^{\prime}$ and $D \subset D^{\prime}$ does not satisfy the altitude formula (Lemma 2.1). Hence $R \subset R^{\prime}$ does not satisfy the altitude formula. Thus $R$ does not satisfy the altitude formula (because if not, $R$ satisfies absolutely the altitude formula and hence the extension $R \subset R^{\prime}$ satisfies the altitude formula). Therefore $R$ is a maximal subring of $\mathrm{qf}(R)$ not satisfying the altitude formula. Thus $R$ is a maximal non-universally catenarian subring of its quotient field, the desired conclusion.

If we leave out the assumption " $T$ is a valuation domain" in the previous proposition, the implication (ii) $\Rightarrow$ (i) fails to be true. (See Example 4.3).

\section{Examples and counterexamples}

This section is concerned with examples showing the limits of the results established in the previous sections.

Example 4.1. For all integers $n, m \in \mathbf{N}$ satisfying $n+1 \leq m \leq$ $2 n+1$, there exists a non-Jaffard local domain $R$ such that $\operatorname{dim} R=n$, $\operatorname{dim} R[X]=m$ and $R$ is a residually integrally closed domain.

If we assume that $m \neq n+1$, then $R$ need not be a maximal nonJaffard subring of its quotient field.

Write $m=n+1+t$. Observe that $0 \leq t \leq n$. Let $K=k\left(X_{1}, X_{2}, \ldots\right)$, where $k$ is a field and $X_{1}, X_{2}, \ldots$ is an infinite number of indeterminates over $k$. Let $Y_{1}, \ldots, Y_{t}, Z_{1}, \ldots, Z_{t}, Z_{t+1}, \ldots, Z_{n}$ be ideterminates over $K$. Let $D_{0}=K$. For $1 \leq j \leq t$, let $D_{j}=D_{j-1}+M_{j}$, where $M_{j}=Z_{j} K\left(Y_{1}, Z_{1}, \ldots, Y_{j-1}, Z_{j-1}, Y_{j}\right)\left[Z_{j}\right]_{\left(Z_{j}\right)}$ is the maximal ideal of the valuation domain $V_{j}=K\left(Y_{1}, Z_{1}, \ldots, Y_{j-1}, Z_{j-1}, Y_{j}\right)\left[Z_{j}\right]_{\left(Z_{j}\right)}$. For $t+1 \leq j \leq n$, let $D_{j}=D_{j-1}+M_{j}$, where $M_{j}=Z_{j} K\left(Y_{1}, Z_{1}, \ldots, Y_{t}, Z_{t}\right.$, $\left.Z_{t+1}, \ldots, Z_{j-1}\right)\left[Z_{j}\right]_{\left(Z_{j}\right)}$ is the maximal ideal of the valaution domain

$$
V_{j}=K\left(Y_{1}, Z_{1}, \ldots, Y_{t}, Z_{t}, Z_{t+1}, \ldots, Z_{j-1}\right)\left[Z_{j}\right]_{\left(Z_{j}\right)} .
$$

Notice that for any $j$ such that $1 \leq j \leq n$, the set of the nonzero prime ideals of $D_{j}$ is $\left\{M_{s}+M_{s+1}+\cdots+M_{j} \mid s=1, \ldots, j\right\}$ which is linearly ordered by inclusion. Observe also that $D_{j}=K+M_{1}+\cdots+M_{j}$.

Let $R=D_{n}, R$ is a local integral domain with maximal ideal $M=$ $M_{1}+\cdots+M_{n}$. We have $\operatorname{dim} R=n, \operatorname{dim} R[X]=m$ and $\operatorname{dim}_{v} R=n+t$ (cf. [1]). The domain $R$ is such that $R / P$ is integrally closed for each $P \in \operatorname{Spec}(R)$. Indeed, $R$ is integrally closed [9, Proposition 2]. Let $P$ be a nonzero prime ideal of $R$, there exists $1 \leq s \leq n$ such that $P=M_{s}+M_{s+1}+\cdots+M_{n}$. We have $R / P=\left(D_{s-1}+M_{s}+\cdots+\right.$ 
$\left.M_{n}\right) /\left(M_{s}+\cdots+M_{n}\right) \simeq D_{s-1}$, which is integrally closed. If we assume that $m>n+1, R$ can not be a maximal non-Jaffard subring of $\mathrm{qf}(R)$. Since if not, then we get $\operatorname{dim}_{v} R=\operatorname{dim} R+1=n+1$ (Theorem 1.4). But $\operatorname{dim} R[X]=m>n+1=\operatorname{dim}_{v} R$, which is impossible.

The next example illustrates the fact that Proposition 2.2 does not hold if $R$ is not integrally closed.

Example 4.2. This example provides a domain $R$ such that:

(a) $R$ is not integrally closed.

(b) $R$ satisfies absolutely the altitude inequality formula.

(c) $R$ is a maximal non-universally catenarian subring of its quotient field.

Let $V, W$ be two incomparable valuation domains with the same quotient field $L$, with maximal ideals $M_{1}$ and $N_{1}$, respectively. Assume that $\operatorname{dim} V=2, \operatorname{dim} W=1$, and that $V / M_{1} \simeq W / N_{1} \simeq K$. Thus $S=V \cap W$ is a semilocal Prüfer domain with maximal ideals $M=M_{1} \cap S$ and $N=N_{1} \cap S$. Set $R:=(S, I, K)$, where $I=M \cap N$. We have $\operatorname{dim} S=2$ and $\operatorname{dim} R=1$ (cf. [9, Corollaire 2]). The integral closure $R^{\prime}$ of $R$ is equal to $S$ [9, Proposition 2]. Hence $R^{\prime}$ is a Prüfer domain. Thus $R$ satisfies absolutely the altitude inequality formula [4, Théorème 2.6].

We have $h t_{S} N+\operatorname{tr} \cdot \operatorname{deg}[S / N: R /(N \cap R)]=1<h t_{R}(N \cap R)+$ tr. $\operatorname{deg}[S: R]=h t_{R} I=2$. Thus the finite type extension $R \subset S$ does not satisfy the altitude formula. Therefore $R$ does not satisfy the altitude formula. Now let $T$ be a domain such that $R \subset T \subseteq \operatorname{qf}(R)$. Our task is to show that $T$ satisfies the altitude formula. We claim that $T^{\prime}$ is a Prüfer domain (since it is an overring of $R^{\prime}$ ). By [14, Proposition 2.6], it will suffices to show that the extension $T \subseteq T^{\prime}$ satisfies the altitude formula. Notice that $T^{\prime}$ is an overring of $S$; then $T^{\prime} \in\left\{S, V, W, V_{Q_{1}^{\prime}}, V_{Q_{1}^{\prime}} \cap W, \operatorname{qf}(S)\right\}([\mathbf{1 6}])$, where $Q_{1}^{\prime}$ is the unique prime ideal of $S$ such that $(0) \subset Q_{1}^{\prime} \subset M$.

Let $Q^{\prime}$ be a nonzero prime ideal of $T^{\prime}$. Set $Q_{1}=Q^{\prime} \cap S, Q=Q^{\prime} \cap T$, and $P=Q^{\prime} \cap R$. Our task is to show that $h t Q^{\prime}=h t Q$. Two cases are then possible:

Case 1: If $Q_{1} \neq N$, then $h t Q_{1}=h t P$. On the other hand, $h t Q^{\prime}=$ $h t Q_{1}$ since the extension $S \subseteq T^{\prime}$ is residually algebraic and satisfies the altitude formula. Thus $h t Q^{\prime}=h t P$. The domain $R$ is locally Jaffard; hence the extension $R \subset T$ satisfies the altitude inequality formula ([4, Théorème 1.5]), in particular, $h t Q \leq h t P$; which yields $h t Q \leq h t Q^{\prime}$. But $T \subset T^{\prime}$ is an integral extension, so it satisfies INC. Thus $h t Q^{\prime} \leq h t Q$. Therefore $h t Q^{\prime}=h t Q$. 
Case 2: If $Q_{1}=N$, then $Q_{1}$ is a maximal ideal of $S$. Hence $Q$ is a maximal ideal of $T^{\prime}$. We discuss the following cases:

(i) If $T^{\prime}=W$, then $T$ is local with $\operatorname{dim} T^{\prime}=\operatorname{dim} T=h t Q^{\prime}=1$. Thus $Q$ is a maximal ideal of $T$. Hence $h t Q^{\prime}=h t Q$.

(ii) The case when either $T^{\prime}=V$ or $T^{\prime}=V_{Q_{1}^{\prime}}$ is impossible since $N$ does not lift in $V$.

(iii) If $T^{\prime}=V_{Q_{1}^{\prime}} \cap W$, then $T^{\prime}$ is semilocal with maximal ideals $Q_{1}^{\prime} T_{Q_{1}^{\prime}}^{\prime}$ and $N T_{N}^{\prime}$. Thus $\operatorname{dim} T^{\prime}=1=h t Q$, and since $\operatorname{dim} T=$ $\operatorname{dim} T^{\prime}=1$, then $h t Q=h t Q^{\prime}=1$.

(iv) If $T^{\prime}=S$, in this case we have the following inclusions $R \subset T \subseteq$ $T^{\prime}=S$. Thus $R / I \subset T / I \subseteq S / I$; that is $K \subset T / I \subseteq K \times K$. Then necessarily we get $T / I=K \times K$. Indeed, $K \times K$ is a $K$ vector space of dimension 2 . Thus $T / I$ is $K$-vector space of dimension at most 2 , and since $T / I \neq K$, then $T / I=K \times K$. Therefore $T=S=T^{\prime}$ and $Q^{\prime}=Q$.

As stated earlier, if we leave out the assumption " $T$ is a valuation domain" in Theorem 3.4, the following example shows, among other facts that the implication (ii) $\Rightarrow$ (i) fails.

Example 4.3. Denote by $D$ the domain $R$ constructed in Example 4.2. Let $L=\mathrm{qf}(D)$ and $X, Y$ be two indeterminates over $L$. Set $V_{1}=$ $L(Y)+X L(Y)[X]_{(X)}$ and $V_{2}=L+Y L[Y]_{(Y)}+(X+1) L(Y)[X]_{(X+1)}$. Then $V_{1}$ and $V_{2}$ are two incomparable valuation domains with maximal ideals $M_{1}=X L(Y)[X]_{(X)}$ and $M_{2}=Y L[Y]_{(Y)}+(X+1) L(Y)[X]_{(X+1)}$, respectively. We have $\operatorname{dim} V_{1}=1$ and $\operatorname{dim} V_{2}=2$. The domain $T_{1}=$ $V_{1} \cap V_{2}$ is a semilocal Prüfer domain with maximal ideals $M^{\prime}=M_{1} \cap T_{1}$ and $N^{\prime}=M_{2} \cap T_{1}$. Let $M=M^{\prime} \cap N^{\prime}$ and $T:=\left(T_{1}, M, L\right)$. Let $R:=(T, M, D)$. Then we have the following properties:

$(\alpha) D$ is a Jaffard domain which is a maximal non-universally catenarian subring of its quotient field. (See Example 4.2).

( $\beta$ ) $T^{\prime}$ is a Prüfer domain since $T^{\prime}=T$.

( $\gamma) D \subset T / M$ is an algebraic extension.

$(\delta) T$ does not satisfy the altitude formula (because the finite type extension $T \subset T_{1}$ does not satisfy the altitude formula). Hence $R$ is not a maximal non-universally catenarian subring of its quotient field. 


\section{References}

[1] D. F. Anderson, A. Bouvier, D. E. Dobbs, M. Fontana AND S. KabBAJ, On Jaffard domains, Exposition. Math. 5 (1988), $145-175$.

[2] D. F. Anderson And D. E. Dobbs, Pairs of rings with the same prime ideals, Canad. J. Math. 32(2) (1980), 362-384.

[3] D. F. Anderson, D. E. Dobbs, S. Kabbaj And S. B. Mullay, Universally catenarian domains of $D+M$ type, Proc. Amer. Math. Soc. 104(2) (1988), 378-384.

[4] A. Ayache And P.-J. CAhen, Anneaux vérifiant absolument l'inégalité ou la formule de la dimension, Boll. Un. Mat. Ital. B (7) 6(1) (1992), 39-65.

[5] A. Ayache, P.-J. CAhen And O. ECHI, Intersection de produits fibrés et formule de la dimension, Comm. Algebra 22(9) (1994), 3495-3509.

[6] A. Ayache, P.-J. Cahen and O. Echi, Anneaux quasi-Prüferiens et P-anneaux, Boll. Un. Mat. Ital. B (7) 10(1) (1996), 1-24.

[7] M. Ben Nasr, O. Echi, L. Izelgue and N. Jarboui, Pairs of domains where all intermediate domains are Jaffard, J. Pure Appl. Algebra 145(1) (2000), 1-18.

[8] A. Bouvier, D. E. Dobbs and M. Fontana, Universally catenarian integral domains, Adv. in Math. 72(2) (1988), 211-238.

[9] P.-J. Cahen, Couples d'anneaux partageant un idéal, Arch. Math. 51(6) (1988), 505-514.

[10] P.-J. CAhen, Construction $(B, I, D)$ et anneaux localement ou residuellement de Jaffard, Arch. Math. 54(2) (1990), 125-141.

[11] D. E. DobBs, Coherence, ascent of going-down and pseudovaluation domains, Houston J. Math. 4(4) (1978), 551-567.

[12] D. E. DobBs, On the weak global dimension of pseudo-valuation domains, Canad. Math. Bull. 21(2) (1978), 159-164.

[13] D. E. Dobbs and M. Fontana, On pseudo-valuation domains and their globalizations, Meeting on "Commutative Algebra", Trento (1981).

[14] O. Echi, Sur les hauteurs valuatives, Boll. Un. Mat. Ital. B (7) 9(2) (1995), 281-297.

[15] M. Fontana, Topologically defined classes of commutative rings, Ann. Mat. Pura Appl. (4) 123 (1980), 331-355.

[16] R. Gilmer, "Multiplicative ideal theory", Pure and Applied Mathematics 12, Marcel Dekker, Inc., New York, 1972. 
[17] J. R. Hedstrom and E. G. Houston, Pseudo-valuation domains, Pacific J. Math. 75(1) (1978), 137-147.

[18] J. R. Hedstrom and E. G. Houston, Pseudo-valuation domains, II, Houston J. Math. 4(2) (1978), 199-207.

[19] I. Kaplansky, "Commutative rings", Revised edition. The University of Chicago Press, Chicago, Ill.-London, 1974.

[20] S. Malik and J. L. Mott, Strong $S$-domains, J. Pure Appl. Algebra 28(3) (1983), 249-264.

[21] S. Visweswaran, Intermediate rings between $D+I$ and $K\left[y_{1}, \ldots, y_{t}\right]$, Comm. Algebra 18(2) (1990), 309-345.

[22] A. R. WADsworth, Pairs of domains where all intermediate domains are Noetherian, Trans. Amer. Math. Soc. 195 (1974), 201-211.

Department of Mathematics

Faculty of Sciences

University of Sfax

3038 Sfax

Tunisia

E-mail address: Mabrouk.Ben.Nasr@fss.rnu.tn

E-mail address: Noomen.Jarboui@fss.rnu.tn

Primera versió rebuda el 9 de febrer de 1999,

darrera versió rebuda el 8 de juliol de 1999. 